\title{
Finnischer Grundwortschatz für Fortgeschrittene
}

Harald Molan: Grundwortschatz Finnisch. Hamburg: Buske 2010. 264 S. Zusätzlich im Internet (http://www.buske.de/download/ 020570.pdf): Finnisches Wörterverzeichnis, $24 \mathrm{~S}$.

Für die in Deutschland an Schulen unterrichteten Fremdsprachen ist das Hilfsmittel des Grund- und Aufbauwortschatzes schon lange etabliert, und in den letzten Jahren findet es sich im Programm des Hamburger Buske-Verlags auch für einige weniger häufig unterrichtete Sprachen. Der 2010 in dieser Reihe erschienene „Grundwortschatz Finnisch" von Harald Molan präsentiert den Wortschatz in 16 verschiedenen Rubriken (von Allgemeines und Zeit über z. B. Haus + Garten; In der Stadt oder Unterwegs bis hin zu Medien; Sport, Freizeit + Kultur; Gesellschaft, Staat + Politik sowie abschließend Grammatikalische Begriffe). Diese enthalten ihrerseits zwischen zwei und elf Unterrubriken. Insgesamt sind die Einträge so in über hundert Themengebiete eingeteilt. Die Zuordnung von Lexemen zu einzelnen Rubriken ist nicht immer ganz intuitiv, anhand des enthaltenen deutsch-finnischen Registers sind Begriffe aber leicht auffindbar. Zum Nachschlagen finnischer Wörter lässt sich das Buch allerdings nur mit Hilfe der auf den Internetseiten des Verlages zu findenden finnisch-deutschen Wortliste nutzen. Leider wurden diese 24 Seiten nicht in das Buch integriert.

Die Fülle von Themengebieten und die im Vorwort genannte Zahl von „etwa 9000 Stichwörter[n]“ (S. 5) zeigt bereits, dass nicht nur der im Titel genannte Grundwortschatz behandelt wird. Der Verfasser bemerkt hierzu selbst, dass auch „Aufbauwortschatz und umgangssprachliche Ausdrücke" berücksichtigt worden seien, wobei der Schwerpunkt auf „Wörter[n] mit Bezug zu Finnland“ gelegen habe (ebd.). Der Autor hat diese Aufgabe ernst genommen: Über den behandelten Wortschatz, die verwendeten Beispiele sowie Erläuterungen und Hinweise erhält der Lerner Einblick in die Besonderheiten der finnischen Sprache, Kultur und Natur. Hierbei wird zwar auch das eine oder andere Klischee bedient (wie Schweigsamkeit oder Trinkfreude), insgesamt ist die Darstellung aber 
sehr gelungen. Speziell „finnische“ Unterrubriken sind z. B. Finnische Spezialitäten in der Hauptrubrik Speisen+Getränke, Wildtiere in Finnland in der Hauptrubrik Natur oder In Lappland in der Hauptrubrik Auf Reisen. In dieser Unterrubrik wird u. a. auf die verschiedenen Bezeichnungen für die Bewohner Lapplands eingegangen (saamelainen, lappalainen, lappilainen; S. 158).

In Bezug auf sprachliche Besonderheiten ist positiv anzumerken, dass der für deutsche Muttersprachler oft schwierigen Unterscheidung der Vokal- und Konsonantenquantität besondere Beachtung geschenkt wird - die Unterschiede werden sowohl in Beispielsätzen aufgegriffen als auch in eigenen Hinweisen auf Verwechslungsmöglichkeiten. So spielt z. B. der Beispielsatz zu punainen 'rot' mit der Vokallänge: „Mikä tämän taideteoksen nimi on? Se on "punainen puunainen ". Wie heißt dieses Kunstwerk? Es heißt »Die rote Baumfrau«." (S. 12), und bei der Einführung von tuli 'Feuer' geht der Autor u. a. auf die Wichtigkeit der Quantität zur Unterscheidung dieses Lexems von tuuli 'Wind' und tulli 'Zoll' ein (S. 110).

Trotz dieser Berücksichtigung der speziellen Bedürfnisse deutscher Lernender ist das vorliegende Buch für die Zielgruppe, die sich durch den Titel des Werkes beson- ders angesprochen fühlen dürfte, also für Anfänger oder Lernende mit wenig Vorkenntnissen, nur unter Vorbehalt zu empfehlen. Ein grundlegendes Problem liegt darin, dass der Autor bewusst auf eine Unterscheidung von Grund- und Aufbauwortschatz verzichtet hat (S. 5). Als Begründung wird angeführt, der Lerner solle selbst bestimmen können, welche Wörter für ihn wichtig oder weniger wichtig seien (ebd.). Abgesehen davon, dass man, um diese Entscheidung treffen $\mathrm{zu}$ können, die Rubriken zunächst einmal ganz durchlesen muss, scheint diese Vorgehensweise gerade für Anfänger nicht unbedingt empfehlenswert: Zwar haben auch sie besondere Interessen, so dass sie in einzelnen Themengebieten möglichst viele Wörter lernen wollen. Auch in den für sie weniger interessanten Bereichen möchten sie sich jedoch in der Regel die häufigsten Wörter aneignen. Statt der teilweise sehr assoziativen Anordnung der Wörter (vgl. z. B. die Abfolge der Lexeme in der Rubrik Der Mensch, Unterrubrik Der Körper (S. 31): ..., vartalo 'Figur, Körper', seksikäs 'sexy', ihminen 'Mensch', mies 'Mann', nainen 'Frau', iho 'Haut', tatuoida 'tätowieren', tatuointi 'Tätowierung', pää 'Kopf'...) wäre eine Abfolge, in der in den thematischen Rubriken zunächst die Wörter des Grundwortschatzes genannt würden und 
dann, ggf. von diesen abgesetzt, diejenigen des Hauptwortschatzes, für Anfänger eher angemessen.

Es wird jedoch nicht nur auf eine Unterscheidung von Grundund Aufbauwortschatz verzichtet, viele Wörter des Grundwortschatzes werden gar nicht oder nicht systematisch eingeführt. So werden z. B. die Personalpronomina nicht zusammenhängend behandelt, sondern finden sich nur in Beispielsätzen oder sporadisch bei der Behandlung spezieller Aspekte (z. B. fehlendes Genus (hän 'er, sie', S. 83)) und schließlich (ohne Übersetzung) in den Flexionstabellen des Anhangs. Eine Einführung hätte zum Beispiel in der Rubrik Allgemeines erfolgen können oder in einer eigenen, in anderen entsprechenden Werken oft anzutreffenden Rubrik Strukturwörter, in der auch das jetzt nur in Beispielsätzen auftauchende und selbst in den Tabellen zur Flexion der Pronomen fehlende Relativpronomen joka, Konjunktionen usw. ihren Platz finden könnten. Den Prä- und Postpositionen ist zwar eine eigene Unterrubrik gewidmet, allerdings handelt es sich hier um die allerletzte Unterrubrik des Buches.

Ein weiterer Punkt, der gerade für Anfänger problematisch sein könnte, ist die oft eher freie Übersetzung von Beispielsätzen, vgl. z. B. „Tilauksesi on saapunut. Se on varattu nimelläsi viikon. Ihre Bestellung ist eingetroffen. Sie ist eine Woche lang auf Ihren Namen reserviert.“ (S. 87). Zwar wird an anderer Stelle erläutert, dass Duzen in Finnland üblicher ist als in Deutschland (vgl. S. 48), für Anfänger ist die augenscheinliche Bevorzugung sinngemäßer, im jeweiligen Kontext äquivalenter Entsprechungen gegenüber exakten, wörtlichen Übersetzungen jedoch zuweilen mühsam. Die Entschlüsselung der genauen Bedeutung der einzelnen Wörter eines Beispielsatzes wird für sie dadurch noch erschwert, dass der Autor für die Beispiele bewusst nicht nur Wörter des Grundwortschatzes verwendet, um „dem Lernenden die Möglichkeit [zu] geben, Wörter selbst in einem Wörterbuch nachzuschlagen und ihr Beugungsmuster zu erahnen." (S. 5).

In einem Grundwortschatz erwartet man im Allgemeinen keine Erläuterungen zur Grammatik. Auch der dem vorliegenden Werk beigefügte Anhang scheint nicht als solche gemeint zu sein - hier finden sich lediglich Anmerkungen zu Alphabet und Aussprache sowie zum Stufenwechsel und eine Reihe von Übersichtstabellen zur Nominalund Verbalflexion. Nicht einmal die für das Finnische doch sehr prägende Vokalharmonie wird erwähnt. Die wenigen über die Tabellen hinausgehenden grammatischen Infor- 
mationen sind zudem für den Lerner nur bedingt hilfreich. So heißt es zum Stufenwechsel: „Die Mehrzahl der Nomen und Verben steht in der Grundform in der starken Stufe und wechselt in die schwache Stufe, z. B. hattu $\rightarrow$ hatun. Es gibt aber auch den umgekehrten Wechsel von der schwachen in die starke Stufe: näyte $\rightarrow$ näytteen." (S. 222). Dass hier nicht näher auf die angesprochenen Unterschiede eingegangen wird, ist für einen groben Überblick noch verständlich, es wird jedoch zudem der falsche Eindruck erweckt, bei der Deklination stehe der Nominativ bzw. bei der Konjugation der Infinitiv jeweils in einer Stufe und alle anderen Kasus bzw. Verbformen in der jeweils anderen. So hat der Anhang vor allem den Zweck, die Zuordnung der im Wörterbuchteil enthaltenen Verben $\mathrm{zu}$ sechs Verbklassen durch Flexions- tabellen zu veranschaulichen. Hier hätte eventuell eine Zuordnung dieser Klassen zu den üblicherweise angesetzten Infinitivtypen (wie sie z. B. in der im Literaturverzeichnis angeführten Finnischen Grammatik von Fred Karlsson, die im gleichen Verlag erschienen ist, beschrieben werden) genügt.

Insgesamt ist festzustellen, dass der Titel „Grundwortschatz" irreführend ist und gerade für Lernende ohne Vorkenntnisse noch mehr Struktur und Genauigkeit bei den Übersetzungen wünschenswert wäre. Molans Grundwortschatz Finnisch ist jedoch für fortgeschrittene Lernende ein optisch sehr ansprechendes, häufig auch amüsantes Hilfsmittel zur systematischen Erweiterung der Sprach- und landeskundlichen Kenntnisse.

Monika Schötschel 